CERN-PH-TH/2010-126

SU-ITP-10/20

\title{
Small N=2 Extremal Black Holes in Special Geometry
}

\author{
Anna Ceresole $^{a}$, Sergio Ferrara ${ }^{b, c}$ and Alessio Marrani ${ }^{d}$ \\ ${ }^{a}$ INFN, Sezione di Torino, Via Pietro Giuria 1, 10125 Torino, Italy \\ ${ }^{b}$ Physics Department, Theory Unit, CERN, \\ CH -1211, Geneva 23, Switzerland \\ c INFN - Laboratori Nazionali di Frascati, \\ Via Enrico Fermi 40, I-00044 Frascati, Italy \\ ${ }^{d}$ Stanford Institute for Theoretical Physics, \\ Stanford University, Stanford, CA 94305-4060,USA
}

\begin{abstract}
We provide an intrinsic classification of the large and small orbits for $\mathrm{N}=2$, 4D extremal black holes on symmetric spaces which does not depend on the duality frame used for the charges or on the special coordinates. A coordinate independent formula for the fake superpotential W, which (at infinity) represents the black hole ADM mass, is given explicitly in terms of invariants of the $\mathrm{N}=2$ special geometry.
\end{abstract}




\section{Introduction}

Black hole solutions of (super-)gravity theories with abelian vector and scalar fields, arising at low energy from superstring or M-theory, are presently at the centre of a broadening field of research. In virtue of their attractor behaviour [1, 2], extremal, static, spherically symmetric black holes have been analyzed for N-extended supergravities in various spacetime dimensions, both in their BPS and the non-BPS branch [3, 4].

The main properties of these black holes are encoded in the effective potential of the geodesic action which, in $\mathrm{N}=2$ language, is given by

$$
V_{B H}=|Z|^{2}+\left|D_{i} Z\right|^{2}
$$

where $Z(\varphi, \bar{\varphi} ; q, p)$ is the $\mathrm{N}=2$ central charge [1, 2]. The extrema of this potential with respect to the scalar fields, $\partial_{\varphi} V_{B H}=0$ yield the attractor solutions, while the attractor values of $V_{B H}$ give the corresponding entropies. In turn, the effective potential can be written in terms of a real scalar function $W\left(\varphi^{i}, \bar{\varphi}^{i} ; q, p\right)$ by

$$
V_{B H}=W^{2}+4 g^{i \bar{\jmath}} \partial_{i} W \partial_{\bar{\jmath}} W
$$

$W$ governs the first order flow (BPS) equations for the radial evolution of the complex scalar fields $\varphi^{i}$ and the warp factor $U$ from asymptotic infinity towards the black hole horizon :

$$
U^{\prime}=-e^{U} W, \quad \varphi^{i}=-2 e^{U} g^{i \bar{\jmath}} \partial_{\bar{\jmath}} W .
$$

The superpotential $W$ also encodes many basic characteristics of the extremal black hole, such as the horizon entropy $S_{B H}=\pi W^{2}$, the $A D M$ mass $M_{A D M}=W$ at infinity, and the scalar charges at infinity, $\Sigma_{i}=\partial_{i} W$. For BPS solutions, $W$ is given by the modulus of the central charge $|Z|$ according to (1.1) [1]. The BPS flows end at the critical points of the central charge $D_{i} Z=\left(\partial_{i}+\frac{1}{2} \partial_{i} K\right) Z=0$, which are also critical point of the full potential (1.2). However [5], the same set up for the non BPS branch [3] requires that one identifies a fake superpotential $W(\varphi, \bar{\varphi} ; q, p)$ whose extrema now describe the non supersymmetric attractors. Therefore, full solutions are more readily obtained from first order BPS-like flow equations as in the supersymmetric case. For these reasons, computing $\mathrm{W}$ has been recently a topical issue.

It has been known for some time that the U-duality of the underlying supergravity dictates many important features of the solutions [6]. This is perfectly illustrated by the maximally extended $N=8$ theory, where there are 70 scalar fields spanning the symmetric space $E_{7(7)} / S U(8)$ and the pointlike electric-magnetic charges form a 56-dimensional vector $Q^{a}$ of the U-duality group $E_{7(7)}$. The area of the horizon for both $1 / 8$-BPS and non-BPS 
attractors is proportional to $\sqrt{ \pm I_{4}}$, where $I_{4}=T_{a b c d} Q^{a} Q^{b} Q^{c} Q^{d}$ is the $E_{7(7)}$ quartic invariant, while a positive or negative sign in $I_{4}$ tells apart a BPS from a non-BPS attractor [7]. More generally, it was shown in [8] that for a generic U-duality group G in extended supergravity, different supersymmetry features of the p-brane solutions can be characterized by some G-invariant conditions on the central charges. For fixed values of the $I_{4}$ invariant in $4 \mathrm{~d}$ and of analogous cubic invariants $I_{3}$ existing in $5 \mathrm{~d}$, the charge vector $\mathrm{Q}$ for supergravity theories based on symmetric spaces describes orbits whose nature determines the amount of supersymmetry preserved by the attractor points [9]. This leads to a classification of the attractors in terms of orbits of the charge vector $\mathrm{Q}$ [10, 11].

Orbits subdivide into regular or "Large", and singular or "Small". Small orbits arise for vanishing horizon area, when $I_{4}=0$, they have zero entropy and they correspond to solutions with no attractor behaviour: the scalars fields never reach a fixed point at finite distance in moduli space. Although the corresponding black hole solutions are singular, they have played an increasingly central role in recent literature because of their relation to the issue of finiteness of $\mathrm{N}=8$ supergravity [12]. Small Orbits were initially examined in [9], then further discussed in [10, 13] while in [14] they have been recently defined by the limit $I_{4} \rightarrow 0$ of large orbits.

Beside the classification of large and small orbits, duality has also played a major role in finding in some generality the fake superpotential $W$, which at first was only known within particular models [5, 15, 16, 17, 4]. To begin with, the $N=2$ central charge is a symplectic product of the symplectic sections with the electric-magnetic charge vector $Q=\left(p^{\Lambda}, q_{\Lambda}\right): Z=$ $e^{K / 2}\left(q_{\Lambda} X^{\Lambda}-p^{\Lambda} F_{\Lambda}\right)$. Also the effective potential itself (1.1) is a symplectic invariant quantity, and the supersymmetric flow equations (1.3) are also driven by the invariant quantity $|Z|$. Therefore, it is reasonable that also the fake superpotential $W$ for the non-BPS branch be built out of symplectic invariants, in agreement with [19] where $W$ is interpreted as the Hamilton's principal function associated to the non-BPS flow equations. A constructive strategy together with an explicit formula for $\mathrm{W}$ in terms of duality invariants was given in [21] for the $t^{3}$ model and more generally in [22] for the $s t^{2}$ and $s t u$ models, also in relation with the $N=8$ theory in the alternative approach of nilpotent orbits [23].

In this note we elucidate the properties of the singular black hole solutions in four dimensional $N=2$ supergravity coupled to $n$ vector multiplets, where the geometry of moduli space is given by special geometry[18] and the duality is encoded in $S p(2 n+2)$ symplectic transformations. Interestingly, although singular solutions have zero entropy and no attractor behaviour, one can still define W[14], which has a runaway behaviour and gets stabilized only at the boundary of moduli space, by taking an $I_{4} \rightarrow 0$ limit of the $W$ for large orbits [14. We will then provide explicit forms for the fake superpotential $\mathrm{W}$ for all small orbits 
in $N=2$ symmetric theories. Our goal here is to describe the amount of supersymmetry preserved in each small orbit and to find the relevant fake superpotential $W$ for each of them. After revisiting the large orbits, we intend to use this universal description in terms of invariants also towards the classification of orbits of the $N=2$ charge vector for symmetric special geometries $\mathrm{G} / \mathrm{H}$, extending similar results obtained in [8] for maximally extended theories.

Duality invariant quantities are those that remain unchanged (transform as scalars) under the simultaneous action of the duality group on the charge vector $Q=\left(p^{\Lambda}, q_{\Lambda}\right)$ and on the scalar fields (expressed through the symplectic sections $\left(X^{\Lambda}, F_{\Lambda}\right)$, with $\left.\Lambda=(0, i)=0, \ldots, n\right)$. Here we recall that the complete set of local $\mathrm{H}$ invariants in $N=2$ special geometry found in [20] is given by

$$
\begin{array}{rlrl}
i_{1} & =Z \bar{Z} & \\
i_{2} & =g^{i \bar{\jmath}} Z_{i} \bar{Z}_{\bar{\jmath}} & \left(Z_{i}=D_{i} Z, \bar{Z}_{\bar{\imath}}=\bar{D}_{\bar{\imath}} \bar{Z}\right), \\
i_{3}=\frac{1}{6}\left[Z N_{3}(\bar{Z})+\overline{Z N}_{3}\left(Z_{i}\right)\right], & i_{4}=\frac{i}{6}\left[Z N_{3}(\bar{Z})-\overline{Z N}_{3}(Z)\right], \\
i_{5}=g^{i \bar{\imath}} C_{i j k} C_{\bar{\imath} \bar{\jmath} \bar{k}} \bar{Z}^{j} \bar{Z}^{k} Z^{\bar{\jmath}} Z^{\bar{k}}, & &
\end{array}
$$

where the cubic norms are given by

$$
N_{3}(\bar{Z})=C_{i j k} \bar{Z}^{i} \bar{Z}^{j} \bar{Z}^{k}, \quad \bar{N}_{3}(Z)=C_{\bar{\imath} \bar{\jmath} \bar{\imath}} Z^{\bar{\imath}} Z^{\bar{\jmath}} Z^{\bar{k}}
$$

These five invariants in the case of symmetric special geometries are not unrelated, and although each one of them depends on the scalar fields and the charges, they satisfy a constraint which involves the quartic $\mathrm{G}$ invariant $I_{4}$ which is field independent:

$$
I_{4}=\left(i_{1}-i_{2}\right)^{2}+4 i_{4}-i_{5}, \quad \partial_{\varphi} I_{4}=0, \quad \partial_{\bar{\varphi}} I_{4}=0
$$

The objects $\left(i_{1}, \ldots, i_{5}\right)$ behave as scalar functions of the charges and the scalar fields under duality transformations.

Our main interest is to compute the superpotential $\mathrm{W}$, as its value at radial infinity gives the ADM mass of the given black hole. In extended supergravity, the BPS bound states that

$$
M_{A D M}=W\left(\varphi_{\infty}, Q\right) \geq\left|z_{h}\right|
$$

where $z_{h}$ is the highest skew eigenvalue of the central charge $Z_{A B}$, which is saturated for BPS solutions. In the $\mathrm{N}=8$ case, an interesting bound holds for the non-BPS orbits [12, 14]

$$
\left|z_{h}\right|^{2}<W_{\text {nonBPS }}^{2} \leq 4\left|z_{h}\right|^{2}
$$


since $W^{2} \leq V_{B H} \leq 4\left|z_{h}\right|^{2}$. In the $N=2$ theory, $Z_{A B}=\epsilon_{A B} Z(A, B=1,2)$ and the highest eigenvalue coincides with the $\mathrm{N}=2$ central charge.

According to the procedure used for the $N=8$ theory in [24], where the four skew eigenvalues of the central charge were put in correspondence with the four eigenvalues of a quartic polynomial, and following the reasonings of [22], one can establish a correspondence between the basis $\left(i_{1}, \ldots, i_{5}\right)$ and the set $\left(i_{1}, \lambda_{1}, \lambda_{2}, \lambda_{3}, I_{4}\right)$ where $\lambda_{i}$ are the roots of a universal cubic equation

$$
\lambda^{3}-i_{2} \lambda^{2}+\frac{i_{5}}{4} \lambda-\frac{i_{3}^{2}+i_{4}^{2}}{4 i_{1}}=0
$$

with real, positive roots given by

$$
\begin{aligned}
& \lambda_{1}=\frac{1}{3}\left(i_{2}+2 \operatorname{Re} w\right), \\
& \lambda_{2}=\frac{1}{3}\left(i_{2}-\operatorname{Re} w-\sqrt{3} \operatorname{Im} w\right), \\
& \lambda_{3}=\frac{1}{3}\left(i_{2}-\operatorname{Re} w+\sqrt{3} \operatorname{Im} w\right) .
\end{aligned}
$$

where

$$
\begin{aligned}
v & =2 i_{2}^{3}+\frac{27\left(i_{3}^{2}+i_{4}^{2}\right)}{4 i_{1}}-\frac{9 i_{2} i_{5}}{4} \\
z & =\frac{9 i_{2}\left(i_{3}^{2}+i_{4}^{2}\right) i_{5}}{8 i_{1}}+\frac{i_{2}^{2} i_{5}^{2}}{16}-\frac{i_{2}^{3}\left(i_{3}^{2}+i_{4}^{2}\right)}{i_{1}}-\frac{27\left(i_{3}^{2}+i_{4}^{2}\right)^{2}}{16 i_{1}^{2}}-\frac{i_{5}^{3}}{16}, \\
w & =\left(\frac{v+3 i \sqrt{3 z}}{2}\right)^{1 / 3} .
\end{aligned}
$$

Therefore $V_{B H}=i_{1}+i_{2}=i_{1}+\lambda_{1}+\lambda_{2}+\lambda_{3}$. In a generic situation, the highest root of the cubic is $\lambda_{1}$ and for small black holes it will coincide with the fake superpotential $W_{\text {nonBPS }}$ in various non-BPS orbits to be discussed below.

We start by giving a characterization of the attractors and the large orbits purely in terms of the invariants (1.4)-(1.7), together with their W superpotential. For the BPS branch, we always have $W^{2}=i_{1}$, while in the non BPS case it has to be determined case by case. We shall see that the only branch where $\mathrm{W}$ is not given by simple radicals is when $I_{4}<0$. The black hole attractors in $\mathrm{N}=2$ theories are defined as solutions of the equation [2]

$$
\partial_{i} V_{B H}=2 \bar{Z} Z_{i}+i C_{i j k} \bar{Z}^{j} \bar{Z}^{k}=0 .
$$

According to the classification of [26], symmetric special geometries subdivide into various series. The first is given by the four Magic supergravities or irreducible Jordan models 
(based on Jordan algebras over $\mathbb{O}, \mathbb{H}, \mathbb{C}, \mathbb{R}[25]$ ). The corresponding BPS attractor point, with $Z_{i}=0$ is given by

$$
\text { BPS : } \quad i_{2}=i_{3}=i_{4}=i_{5}=0, \lambda_{1}=\lambda_{2}=\lambda_{3}=0, \quad I_{4}=i_{1}^{2}, \quad W=\sqrt{i_{1}} .
$$

They also admit a non BPS attractor point for $Z_{i} \neq 0$ and $Z=0$, which is given by

$$
\text { non BPS, } Z=0: \quad i_{1}=i_{3}=i_{4}=i_{5}=0 ; \quad \lambda_{1}=i_{2}, \quad \lambda_{2}=\lambda_{3}=0 ; \quad I_{4}=i_{2}^{2}, W=\sqrt{i_{2}} \text {. }
$$

Finally, a $Z_{i} \neq 0, Z \neq 0$ nonBPS attractor point occurs at

$$
i_{2}=3 i_{1}, \quad i_{3}=0, \quad i_{4}=-2 i_{1}^{2}, \quad i_{5}=12 i_{1}^{2}
$$

and then at these points

$$
i_{1}=\lambda_{1}=\lambda_{2}=\lambda_{3}, \quad I_{4}=-16 i_{1}^{2}<0, W=2 \sqrt{i_{1}} .
$$

The above attractor points are actually part of three regular orbits, with $I_{4} \neq 0$, which can be characterized as:

$$
\begin{aligned}
& I_{4}>0:\left\{\begin{array}{cc}
\operatorname{BPS} & i_{1}>\lambda_{1}, \lambda_{2}, \lambda_{3} \\
\text { non BPS } & \lambda_{1}>i_{1}, \lambda_{2}, \lambda_{3}
\end{array}\right. \\
& I_{4}<0 \quad \text { non BPS } \quad \lambda_{1} \neq \lambda_{2} \neq \lambda_{3} .
\end{aligned}
$$

While the superpotentials for $I_{4}>0$ are respectively $W_{B P S}=\sqrt{i_{1}}$ and $W_{\text {nonBPS }}=\sqrt{\lambda_{1}}$, for $I_{4}<0$ it has a complicated expression involving radicals that has been found by studying on the $s t^{2}$ and the stu models [22, 23].

Another instance of symmetric special geometry is the infinite reducible series,

$$
N=2: \quad \frac{G}{H}=\frac{S L(2, \mathbb{R})}{U(1)} \times \frac{S O(2, n)}{S O(2) \times S O(n)}
$$

where due to the factorization of the moduli space $Z_{i}=Z_{s}, Z_{I}$ and the basic cubic equation has factorized eigenvalues $i_{s}, \lambda_{1}, \lambda_{2}$ where $i_{s}=Z_{s} \bar{Z}_{s}$. The BPS attractor point is

$$
i_{s}=0, \quad \lambda_{1}=\lambda_{2}=0, \quad I_{4}=i_{1}^{2}
$$

while the non BPS attractor points for $I_{4}>0$ are

$$
Z=0: \quad W=\sqrt{i_{s}}, \quad i_{1}=\lambda_{1}=\lambda_{2}=0 \quad I_{4}=i_{s}^{2},
$$




$$
Z=0, Z_{I} Z^{I}=0: \quad W=\sqrt{\lambda_{1}}, \quad i_{1}=i_{s}=\lambda_{2}=0 \quad I_{4}=\left(Z_{I} \bar{Z}^{I}\right)^{2} .
$$

There exist four large orbits:

$$
\begin{aligned}
& I_{4}>0\left\{\begin{array}{lll}
\operatorname{BPS} & i_{1}>i_{s}, \lambda_{1}, \lambda_{2}, & W_{\mathrm{BPS}}=\sqrt{i_{1}} \\
\text { nonBPS } & i_{s}>i_{1}, \lambda_{1}, \lambda_{2}, & W_{\mathrm{nonBPS}}=\sqrt{i_{s}} \\
\text { nonBPS } & \lambda_{1}>i_{1}, i_{s}, \lambda_{2}, & W_{\text {nonBPS }}=\sqrt{\lambda_{1}}
\end{array}\right. \\
& I_{4}<0 \quad \text { nonBPS }
\end{aligned}
$$

where again there is a complicated fake superpotential for $I_{4}<0$ which, however, on the curve $i_{3}=0$, reduces to $W=\frac{1}{2}\left(\sqrt{i_{1}}+\sqrt{\lambda_{1}}+\sqrt{\lambda_{2}}+\sqrt{\lambda_{3}}\right)$ [15, 22, 23].

\section{Small orbits and their W superpotential}

We first consider small orbits for the 4 irreducible Magic supergravities (based on Jordan algebras over $\mathbb{O}, \mathbb{H}, \mathbb{C}, \mathbb{R}[25]$ ) and we impose suitable differential constraints along the lines of [8, 20]. The rank $r$ refers to the minimal number of charges that characterize the given orbit [10].

i) Lightlike orbits

They are simply defined by $I_{4}=0$ and have rank $r=3$. This condition allows to eliminate the invariant $i_{5}$ by posing $i_{5}=\left(i_{1}-i_{2}\right)+4 i_{4}$. The basic cubic equations (1.12) in this case will have three distinct roots, assuming a specific (not particularly illuminating) form upon eliminating $i_{5}$. The BPS orbit will arise when $i_{1}>\lambda_{1}$, while the non-BPS orbit will take place when $i_{1}<\lambda_{1}$. Therefore we will have

$$
I_{4}=0 r=3: \quad \begin{cases}\operatorname{BPS} i_{1}>\sqrt{\lambda_{1}} ; & W_{\mathrm{BPS}}=\sqrt{i_{1}} \\ \operatorname{nonBPS} i_{1}<\sqrt{\lambda_{1}} ; & W_{\text {non BPS }}=\sqrt{\lambda_{1}}\end{cases}
$$

This is also in agreement with the analysis of [14] where this orbit is seen to arise as a limit $I_{4} \rightarrow 0$ of the $I_{4}>0$ non-BPS large orbit.

\section{ii) Critical Orbits}

This second class of orbits is obtained by taking vanishing first derivatives of (1.9) with respect to the central charges $Z$ and their covariant derivatives $Z_{i}$, using the definitions (1.4 1.7). It has rank $r=2$. We find the system of equations 


$$
\begin{aligned}
I_{4} & =\left(i_{1}-i_{2}\right)^{2}+4 i_{4}-i_{5}=0, \\
Z \frac{\partial I_{4}}{\partial Z} & =2\left(i_{1}-i_{2}\right) i_{1}+2\left(i_{4}+i i_{3}\right)=0 \\
Z_{i} \frac{\partial I_{4}}{\partial Z_{i}} & =2\left(i_{2}-i_{1}\right) i_{2}+6\left(i_{4}-i i_{3}\right)-2 i_{5}=0,
\end{aligned}
$$

yielding the conditions

$$
\partial I_{4}=0 \rightarrow\left\{\begin{array}{l}
i_{3}=0 \\
i_{4}=\left(i_{2}-i_{1}\right) i_{1}>0 \\
i_{5}=\left(i_{2}-i_{1}\right)\left(i_{2}+3 i_{1}\right) \quad\left(i_{2}>i_{1}\right) .
\end{array}\right.
$$

For the roots of the basic cubic equation, these constraints on the first derivatives yield

$$
z=0, \quad v=\frac{1}{4}\left(3 i_{1}-i_{2}\right)^{3}, \quad w=\left(\frac{v}{2}\right)^{1 / 3} .
$$

There are two cases according to the possible values of the parameter $v$ :

a) $i_{1}>\frac{i_{2}}{3}, v=v^{*}, w=w^{*}=\frac{3 i_{1}-i_{2}}{2}$, so that $\lambda_{1}=i_{1}$ and $\lambda_{2}=\lambda_{3}=\frac{i_{2}-i_{1}}{2}$

b) $i_{1}<\frac{i_{2}}{3}, v \neq v^{*}, w=\left(\frac{1}{2}+i \frac{\sqrt{3}}{2}\right)\left(\frac{i_{2}-3 i_{1}}{2}\right)$, so that $\lambda_{1}=\frac{i_{2}-i_{1}}{2}, \lambda_{2}=i_{1}$ and $\lambda_{3}=\lambda_{1}$.

The first is the BPS case, where the highest eigenvalue is $\lambda_{1}=i_{1}$, while the second case is the non BPS, where we find $\lambda_{1}=\frac{i_{2}-i_{1}}{2}$. Therefore we get

$$
\partial I_{4}=0, r=2: \begin{cases}\operatorname{BPS} \quad i_{2}>i_{1}>\frac{i_{2}}{3} & W_{\mathrm{BPS}}=\sqrt{i_{1}} \\ & i_{1}=\lambda_{1} ; \lambda_{2}=\lambda_{3}=\frac{i_{2}-i_{1}}{2} \\ \text { nonBPS } \quad i_{1}<\frac{i_{2}}{3} & W_{\text {non BPS }}=\sqrt{\frac{i_{2}-i_{1}}{2}}\end{cases}
$$

iii) Doubly critical orbit

We must consider the projection of the second derivatives on the adjoint representation of $\mathrm{G}, \partial_{A d j}^{2} I_{4}=0$, which, analogously to the $\mathrm{N}=8$ and $\mathrm{N}=4$ theories [8, 20], leads to two second order differential operators on $I_{4}$ that read:

$$
\left(C_{i j k} \frac{\partial^{2}}{\partial Z_{j} \partial Z_{k}}+2 i g_{i \bar{\jmath}} \frac{\partial^{2}}{\partial Z \partial \bar{Z}_{\bar{\jmath}}}\right) I_{4}=0, \quad\left(R_{i \bar{\jmath} k \bar{l}} \frac{\partial^{2}}{\partial Z_{k} \partial \bar{Z}_{\bar{l}}}+2 g_{i \bar{\jmath}} \frac{\partial^{2}}{\partial Z \partial \bar{Z}}\right) I_{4}=0 .
$$

The first one results in an equation like the attractor equation (1.19), but with an opposite relative sign. Together, they yield the extra condition $i_{2}=3 i_{1}$ and thus $i_{4}=2 i_{1}^{2}$ and $i_{5}=12 i_{1}^{2}$. Adding these constraints to the previous ones leads to only one BPS orbit, since one has $i_{1}=\lambda_{1}=\lambda_{2}=\lambda_{3}$. We have

$$
\partial_{A d j}^{2} I_{4}=0, \quad r=1: \quad \operatorname{BPS} \quad i_{1}=\lambda_{1}=\lambda_{2}=\lambda_{3} ; \quad W_{\mathrm{BPS}}=\sqrt{i_{1}}
$$


In this case, the relations among $i_{3}, i_{2}, i_{5}$ are the same as in the $I_{4}<0$ attractor point, but with a flipped sign in $i_{4}$.

To summarize, the generic four Magic models of $\mathrm{N}=2$ symmetric special geometries admit 5 small orbits, defined by three main classes of G-invariant constraints given in terms of the H-invariants $\left(i_{1}, i_{2}, i_{3}, i_{4}, i_{5}\right)$ :

$$
\begin{array}{ll}
\text { 1) } r=3 & i_{5}=\left(i_{1}-i_{2}\right)^{2}+4 i_{4} \\
\text { 2) } r=2 & i_{3}=0 ; \quad i_{4}=i_{1}\left(i_{2}-i_{1}\right)>0 ; \quad i_{5}=\left(i_{2}-i_{1}\right)\left(i_{2}+3 i_{1}\right) \\
\text { 3) } r=1 & i_{2}=3 i_{1} ; i_{3}=0 ; \quad i_{4}=2 i_{1}^{2} ; \quad i_{5}=12 i_{1}^{2} .
\end{array}
$$

Among the allowed symmetric spaces for $N=2$ special geometry classified in [26] there is the infinite cubic sequence (1.26) which describes the coupling of an arbitrary number $n$ of vector multiplets. The factorization of the manifold into two elements requires a separate analysis. However, due to the similarity with its $\mathrm{N}=4$ ancestor

$$
N=4: \quad \frac{G}{H}=\frac{S L(2, \mathbb{R})}{U(1)} \times \frac{S O(6, n)}{S O(6) \times S O(n)}
$$

one can adapt the formalism of [27, 20] to the present case by replacing $Z_{A B}$ by the pair $\left(Z, i \bar{Z}_{s}\right)$, where $s$ is the single modulus of $S L(2) / U(1)$. The unique quartic invariant combination which does not depend on the scalar fields $s, \varphi^{I}$, is given by

$$
I_{4}=S_{1}^{2}-S_{2} \bar{S}_{2}
$$

where now $S_{1}$ and $S_{2}$ must be given by

$$
\begin{aligned}
& S_{1}=|Z|^{2}+\left|Z_{S}\right|^{2}-Z_{I} \bar{Z}^{I} \quad\left(Z_{S}=D_{S} Z, Z_{I}=D_{I} Z\right) \\
& S_{2}=2 i Z \bar{Z}_{s}-Z_{I} Z^{I}
\end{aligned}
$$

Then the quartic invariant (2.9) becomes

$$
I_{4}=\left(Z \bar{Z}-Z_{s} \bar{Z}_{s}-Z_{I} \bar{Z}^{I}\right)^{2}+2 i\left(Z \bar{Z}_{s} \bar{Z}^{I} \bar{Z}_{I}-\bar{Z} Z_{s} Z^{I} Z_{I}\right)-4 Z_{s} \bar{Z}_{s} Z^{I} \bar{Z}_{I}-Z_{I} Z^{I} \bar{Z}_{K} \bar{Z}^{K}
$$

which agrees with (1.9) upon using, for this reducible manifold the invariants

$$
\begin{aligned}
i_{1} & =Z \bar{Z} \quad i_{2}=Z_{s} \bar{Z}_{s}+Z_{I} \bar{Z}^{I} \\
i_{4} & =\frac{i}{2}\left(Z \bar{Z}_{s} \bar{Z}^{I} \bar{Z}_{I}-\bar{Z} Z_{s} Z^{I} Z_{I}\right) \\
i_{5} & =4 Z_{s} \bar{Z}_{s} Z^{I} \bar{Z}^{I}+Z_{I} Z^{I} \bar{Z}_{K} \bar{Z}^{K}
\end{aligned}
$$


The cubic polynomial in this case factorizes as [22]

$$
\begin{aligned}
& \left(\lambda-i_{s}\right)\left(\lambda^{2}-a \lambda+b\right)=0 \\
& i_{s}=Z_{s} \bar{Z}_{s} \quad a=Z_{I} \bar{Z}^{I}=i_{I} \quad b=\frac{1}{4}\left|Z_{I} Z^{I}\right|^{2}
\end{aligned}
$$

which is consistent with the full cubic equation (1.12) as

$$
i_{2}=i_{s}+a=\left|Z_{s}\right|^{2}+Z_{I} \bar{Z}^{I}, \quad \frac{i_{5}}{4}=i_{s} i_{I}+b, \quad \frac{i_{3}^{2}+i_{4}^{2}}{4 i_{1}}=i_{s} b .
$$

Similarly to the Magic supergravities, one can obtain the following classification of orbits (the nomenclature is taken from [20, 14]) with $I_{4}=S_{1}^{2}-S_{2} \bar{S}_{2}=0$ in terms of $S_{1}$ and $S_{2}$ with

$$
S_{1}=i_{1}+i_{s}-i_{I}, \quad\left|S_{2}\right|=\left[i_{5}-4 i_{4}+4 i_{s}\left(i_{1}-i_{I}\right)\right]^{1 / 2}
$$

- Lightlike orbit: $I_{4}=0, r=3$ :

$$
\begin{aligned}
& C_{1}:\left\{\begin{array}{ll}
\operatorname{BPS} & \text { if } i_{1}>i_{s}, \lambda_{1}, \lambda_{2} \\
\text { nonBPS } & \text { if } i_{s}>i_{1}, \lambda_{1}, \lambda_{2}
\end{array} \quad S_{1}>0 \quad \operatorname{Adj}_{S O(2, n)} \neq 0 \quad \operatorname{Adj}_{S L(2)} \neq 0\right. \\
& C_{2}: \operatorname{nonBPS} \quad \lambda_{1}>i_{1}, i_{s}, \lambda_{2} \quad S_{1}<0 \quad \operatorname{Adj}_{S O(2, n)} \neq 0 \quad \operatorname{Adj}_{S L(2)} \neq 0
\end{aligned}
$$

- Critical orbit: $\partial I_{4}=0, r=2$ :

$$
\begin{aligned}
& A_{1}: \mathrm{BPS} \quad i_{1}=i_{s}>\lambda_{1}=\lambda_{2} \quad S_{1}>0 \quad \operatorname{Adj}_{S O(2, n)}=0, \operatorname{Adj}_{S L(2)} \neq 0 \\
& A_{2} \text { : non BPS } \lambda_{1}=\lambda_{2}>i_{1}=i_{s} \quad S_{1}<0 \quad \operatorname{Adj}_{S O(2, n)}=0, \operatorname{Adj}_{S L(2)} \neq 0 \\
& B:\left\{\begin{array}{ll}
\operatorname{BPS} & \text { if } i_{1}=\lambda_{1}>i_{s}=\lambda_{2} \\
\text { non BPS } & \text { if } i_{s}=\lambda_{1}>i_{1}=\lambda_{2}
\end{array} \quad S_{1}=S_{2}=0 \quad \operatorname{Adj}_{S O(2, n)} \neq 0, \operatorname{Adj}_{S L(2)}=0\right.
\end{aligned}
$$

- Doubly critical $\partial_{A d j}^{2} I_{4}=0, r=1$ :

$$
A_{3}: \operatorname{BPS} \quad i_{1}=i_{s}=\lambda_{1}=\lambda_{2} \quad S_{1}=S_{2}=0 \quad \operatorname{Adj}_{S O(2, n)}=\operatorname{Adj}_{S L(2)}=0
$$

We see that reducibility of the manifold leads to a degeneration of orbits that turn out to be 8 rather than 5 of the Magic models case. The splitting of the $\mathrm{B}$ and $C_{1}$ orbits compared to the $N=4$ case are related to the two possible situations $i_{1}>i_{s}$ and $i_{i}<i_{s}$ of the two eigenvalues of $\mathrm{N}=4$ that here are not on the same footing. 
As far as the superpotential $\mathrm{W}$ is concerned, we always have for the supersymmetric orbits in $\mathrm{N}=2 W_{\mathrm{BPS}}=\sqrt{i_{1}}$, while for the non supersymmetric case we have

$$
\begin{cases}B: & W_{\text {nonBPS }}=\sqrt{\lambda_{1}}=\sqrt{i_{s}} ; \\ A_{2}: & W_{\text {nonBPS }}=\sqrt{\lambda_{1}}=\sqrt{\lambda_{2}} ; \\ C_{1}: & W_{\text {nonBPS }}=\sqrt{i_{s}} ; \\ C_{2}: & W_{\text {nonBPS }}=\sqrt{\lambda_{1}} .\end{cases}
$$

Note that the corresponding orbits $B, C_{1}$ in $\mathrm{N}=4$ were $1 / 4 \mathrm{BPS}$ because in that case $i_{s}$ was the second eigenvalue of the central charge and there is a symmetry in the exchange $i_{1} \rightarrow i_{s}$.

To complete the analysis of $\mathrm{N}=2$ symmetric special geometries, one should still consider the quadratic series

$$
\frac{G}{H}=\frac{S U(1, n)}{S U(n) \times U(1)}
$$

having $C_{i j k}=0$ and then $i_{3}=i_{4}=i_{5}=0$. For these values there is only one eigenvalue of the cubic, $\lambda=i_{2}$. This is an interesting case that cannot be derived from five dimensions. The quartic invariant actually becomes quadratic,

$$
\text { Quadratic series: } \quad I_{4}=I_{2}=\left(i_{1}-i_{2}\right)^{2} \text {, }
$$

and one is left with the simple analysis. For the large orbits, one has [10] :

$$
\begin{cases}\mathrm{BPS}: i_{1}>i_{2}, \quad I_{2}>0, & W=\sqrt{i_{1}} \\ \text { non BPS }: i_{1}<i_{2}, \quad I_{2}<0 & W=\sqrt{i_{2}}\end{cases}
$$

with attractor points $i_{2}=0$ and $i_{1}=0$ respectively.

There is only one small orbit, arising for $i_{1}=i_{2}$ everywhere; it has $W=\sqrt{i_{1}}$ and it is BPS.

\section{Summary}

This approach gives a clean intrinsic classification of both large and small orbits of $N=2$ black holes for special geometries based on symmetric spaces $G / H$ entirely in terms of the $\mathrm{H}$ invariants. This allows to make no reference to a particular symplectic frame or to special coordinates. Moreover, this formalism makes it transparent to see where the various BPS conditions come from. As a further outcome, we have determined invariant expressions for the the fake superpotential $W_{\text {non BPS }}=\sqrt{\lambda_{1}}$ for each distinct small non supersymmetric orbit, which turns out to be always given by simple radicals of the highest root of the cubic. The only case where the fake superpotential is not given by a simple radical is the large orbit 
$I_{4}<0$ discussed in [22]. The reason for the calculability of $\mathrm{W}$ on all the small orbits can be understood by looking at the $N=8$ theory, whose truncation to $\mathrm{N}=2$ reproduces almost all the $\mathrm{N}=2$ symmetric models. In $\mathrm{N}=8$, all the small orbits are BPS, as they preserve $1 / 8$, $1 / 4,1 / 2$ supersymmetry. Since the fake superpotential at infinity yields the ADM mass, the BPS bound implies

$$
W_{\infty}>\left\{\left|z_{1}\right|,\left|z_{2}\right|,\left|z_{3}\right|,\left|z_{4}\right|\right\}
$$

where $\left\{z_{i}\right\}$ are the four skew eigenvalues of the central charge matrix $Z_{A B}$. In the $N=2$ truncation, these four eigenvalue split into $\left\{Z, \lambda_{1}, \lambda_{2}, \lambda_{3}\right\}$, where $|Z|^{2}=i_{1}$. Then clearly for small BPS black holes the BPS bound gives $M_{A D M}^{2}=|Z|>\lambda_{1}, \lambda_{2}, \lambda_{3}$, while for small non BPS black holes $M_{A D M}^{2}=\lambda_{1}>|Z|, \lambda_{2}, \lambda_{3}$. Conversely, for the large non BPS black holes $W_{\text {nonBPS }}$ must exceed the BPS bound and it must be greater than any of the eigenvalues $\left\{Z, \lambda_{1}, \lambda_{2}, \lambda_{3}\right\}$. Its expression must be computed by other means [21, 23, 22] and one can see by taking its value at the attractor point that $W_{\text {nonBPS }}=2 \sqrt{i_{1}}\left(i_{1}=\lambda_{1}=\lambda_{2}=\lambda_{3}\right)$ which indeed is double the value of the BPS case.

As a last comment, the relation between invariants $I_{4}$ and $I_{3}$ suggests that one can use this method to invariantly describe the stratification of orbits between five and four dimensions, a task that is left for future work.

\section{Acknowledgments}

This work is supported in part by the ERC Advanced Grant no. 226455, "Supersymmetry, Quantum Gravity and Gauge Fields" (SUPERFIELDS) and in part by MIUR-PRIN contract 20075ATT78 and DOE Grant DE-FG03-91ER40662. The work of A. M. has been supported by an INFN visiting Theoretical Fellowship at SITP, Stanford University, CA, USA.

\section{References}

[1] S. Ferrara, R. Kallosh and A. Strominger, "N=2 extremal black holes," Phys. Rev. D 52 (1995) 5412; A. Strominger, "Macroscopic entropy of $\mathrm{N}=2$ extremal black holes", Phys. Lett. B383, 39 (1996); S. Ferrara and R. Kallosh, "Supersymmetry and attractors", Phys. Rev. D54, 1514 (1996); S. Ferrara, R. Kallosh, "Universality of supersymmetric attractors", Phys. Rev. D 54, 1525 (1996).

[2] S. Ferrara, G. W. Gibbons and R. Kallosh, "Black holes and critical points in moduli space", Nucl. Phys. B500, 75 (1997). 
[3] K. Goldstein, N. Iizuka, R. P. Jena and S. P. Trivedi, "Non-supersymmetric attractors," Phys. Rev. D 72, 124021 (2005) |arXiv:hep-th/0507096]; E. G. Gimon, F. Larsen and J. Simon, "Black Holes in Supergravity: the non-BPS Branch," JHEP 0801 (2008) 040 [arXiv:hep-th/0710.4967].

[4] S. Ferrara, A. Gnecchi and A. Marrani, "d=4 Attractors, Effective Horizon Radius and Fake Supergravity," Phys. Rev. D 78 (2008) 065003 [arXiv:hep-th/0806.3196]; J. Perz, P. Smyth, T. Van Riet and B. Vercnocke, "First-order flow equations for extremal and non-extremal black holes," JHEP 0903 (2009) 150 [arXiv:hep-th/0810.1528]; K. Goldstein and S. Katmadas, "Almost BPS black holes," JHEP 0905 (2009) 058 [arXiv:hepth/0812.4183]; I. Bena, G. Dall'Agata, S. Giusto, C. Ruef and N. P. Warner, "NonBPS Black Rings and Black Holes in Taub-NUT," JHEP 0906 (2009) 015 [arXiv:hepth/0902.4526]; P. Galli and J. Perz, "Non-supersymmetric extremal multicenter black holes with superpotentials," arXiv:0909.5185.

[5] A. Ceresole and G. Dall'Agata, "Flow Equations for Non-BPS Extremal Black Holes," JHEP 0703 (2007) 110 arXiv:hep-th/0702088.

[6] C. M. Hull and P. K. Townsend, Nucl. Phys. B438 109 (1995); M. Cvetic and C. M. Hull, "Black holes and U-duality," Nucl. Phys. B 480 (1996) 296 arXiv:hep-th/9606193.

[7] R. Kallosh and B. Kol, "E(7) Symmetric Area of the Black Hole Horizon," Phys. Rev. D 53, 5344 (1996) arXiv:hep-th/9602014.

[8] S. Ferrara and J. M. Maldacena, "Branes, central charges and U-duality invariant BPS conditions," Class. Quant. Grav. 15, 749 (1998) arXiv:hep-th/9706097.

[9] S. Ferrara and M. Gunaydin, "Orbits of exceptional groups, duality and BPS states in string theory", Int. J. Mod. Phys. A13 (1998) 2075.

[10] S. Bellucci, S. Ferrara, M. Gunaydin and A. Marrani, "Charge orbits of symmetric special geometries and attractors," Int. J. Mod. Phys. A 21 (2006) 5043 arXiv:hep-th/0606209.

[11] L. Andrianopoli, R. D'Auria and S. Ferrara, "U-duality and central charges in various dimensions revisited," Int. J. Mod. Phys. A 13, 431 (1998) arXiv:hep-th/9612105;

L. Andrianopoli, R. D'Auria and S. Ferrara, "Five dimensional U-duality, black-hole entropy and topological invariants," Phys. Lett. B 411, 39 (1997) arXiv:hep-th/9705024. 
[12] M. B. Green, H. Ooguri and J. H. Schwarz, "Decoupling Supergravity from the Superstring," Phys. Rev. Lett. 99 (2007) 041601 [arXiv:0704.0777 [hep-th]]; M. Bianchi, S. Ferrara and R. Kallosh, "Observations on Arithmetic Invariants and U-Duality Orbits in N =8 Supergravity," JHEP 1003, 081 (2010) [arXiv:0912.0057 [hep-th]]; "Perturbative and Non-perturbative N =8 Supergravity," arXiv:0910.3674 [hep-th], to appear on Phys. Lett. B.

[13] L. Andrianopoli, R. D'Auria, S. Ferrara and M. Trigiante, "Extremal Black Holes in Supergravity", Lect.Notes Phys.737:661 (2008), arXiv:hep-th/0611345

[14] L. Andrianopoli, R. D'Auria, S. Ferrara and M. Trigiante, "Fake Superpotential for Large and Small Extremal Black Holes," arXiv:1002.4340 [hep-th].

[15] L. Andrianopoli, R. D'Auria, E. Orazi and M. Trigiante, "First Order Description of Black Holes in Moduli Space," JHEP 0711 (2007) 032 [arXiv:hep-th/0706.0712].

[16] S. Bellucci, S. Ferrara, A. Marrani and A. Yeranyan, "stu Black Holes Unveiled", Entropy 10, (2008) 507 [arXiv:hep-th/0807.3503].

[17] G. Lopes Cardoso, A. Ceresole, G. Dall'Agata, J. M. Oberreuter and J. Perz, "Firstorder flow equations for extremal black holes in very special geometry," JHEP 0710 (2007) 063 [arXiv:hep-th/0706.3373].

[18] A. Strominger, "Special Geometry," Commun. Math. Phys. 133 (1990) 163.

[19] L. Andrianopoli, R. D'Auria, E. Orazi and M. Trigiante, "First Order Description of $\mathrm{D}=4$ static Black Holes and the Hamilton-Jacobi equation," [arXiv:hep-th/0905.3938].

[20] B. L. Cerchiai, S. Ferrara, A. Marrani and B. Zumino, "Duality, Entropy and ADM Mass in Supergravity," Phys. Rev. D 79 (2009) 125010 [arXiv:0902.3973].

[21] A. Ceresole, G. Dall'Agata, S. Ferrara and A. Yeranyan, "First order flows for $\mathrm{N}=2$ extremal black holes and duality invariants," Nucl.Phys. B824:239-253,2010 arXiv:0908.1110 .

[22] A. Ceresole, G. Dall'Agata, S. Ferrara and A. Yeranyan, "Universality of the superpotential for d=4 extremal black holes", Nucl. Phys. B832 (2010) 358, arXiv:0910.2697.

[23] G. Bossard, Y. Michel and B. Pioline, "Extremal black holes, nilpotent orbits and the true fake superpotential," JHEP 1001, 038 (2010) [arXiv:0908.1742 [hep-th]]. 
[24] R. D'Auria, S. Ferrara and M. A. Lledo, "On central charges and Hamiltonians for 0-brane dynamics," Phys. Rev. D 60 (1999) 084007 arXiv:hep-th/9903089].

[25] M. Gunaydin, G. Sierra and P. K. Townsend, "The Geometry Of N=2 Maxwell-Einstein Supergravity And Jordan Algebras," Nucl. Phys. B 242 (1984) 244.

[26] E. Cremmer and A. Van Proeyen, "Classification of Kähler Manifolds in N = 2 Vector Multiplet Supergravity Couplings", Class. Quantum Grav.2, 445 (1985); B. de Wit, F. Vanderseypen and A. Van Proeyen, "Symmetry Structures of Special Geometries", Nucl. Phys. B400, 463 (1993), hep-th/9210068.

[27] L. Andrianopoli, R. D'Auria and S. Ferrara, "U-invariants, black-hole entropy and fixed scalars," Phys. Lett. B 403 (1997) 12 [arXiv:hep-th/9703156]. 